\title{
THE EQUATIONS OF THE TECHNICAL THEORY OF SHELLS WITH THE EFFECT OF TRANSVERSE SHEAR DEFORMATION*
}

\author{
BY
}

\author{
STANISŁAW A. ŁUKASIEWICZ
}

Warsaw

Introduction. The theory of shallow shells in its original form was worked out by Donnell [1], Musthari [2], Marguerre [3] and Vlasov [4]. Due to their simple construction, their equations are useful for numerical calculations of definite technical problems. Their further virtue is that they become the equations of plates when the curvature of the shell decreases to zero, but the results obtained by means of this theory are accurate enough only for the case of shallow shells. More exact equations can be obtained by the introduction of certain small improvements [5]. The difference in the derivation of the Donnell-Vlasov equations and the new ones is the following, among others. In deriving the Donnell-Vlasov equations small terms containing principal and Gaussian curvatures as factors have been neglected. In developing the new equations we need neglect only some small terms containing the derivatives of the curvatures. The improved equations are already quite satisfactory for technical purposes. The accuracy for a shell of positive and slowly varying curvature is of the order of 1 to 2 per cent. The purpose of the present paper is to introduce into the above equations the effect of transverse shear deformation and the effect of transverse normal stresses. In this way we obtain improved simple equations allowing a somewhat more exact analysis of the behavior of shells under concentrated loads, for example. Papers in which the effects of both transverse normal stress and shear deformation have been accounted for include those by Hildebrand, Reissner and Thomas [6], Green and Zerna [7] and Reissner [8], [9]. Equations of the linear theory of shallow shells which include the effect of transverse shear deformation have been obtained by Naghdi [10]. A second work by Naghdi [11] is concerned with the formulation of stress-strain relations and appropriate boundary conditions in the theory of small deformations of thin shells. Wilkinson and Kalnins considered in [12] and [13] the case of a spherical shell loaded by a normal concentrated dynamic force while taking into account the effect of transverse shear deformations. Improved equations for the spherical shell were obtained in [12].

In what follows we develop the equations of the theory of shells of slowly varying curvature, taking into account the effect of transverse shear deformation and transverse normal stress.

1. Geometry and deformation of the shell. Let us consider an isotropic shell of constant thickness and apply a system of orthogonal curvilinear coordinates $\left(\alpha_{1}, \alpha_{2}\right)$ whose directions follow the directions of the principal curvatures of the shell surface.

* Received October 17, 1968; revised version received November 10, 1969. 
Let us assume the third coordinate $\alpha_{3}=z$ to be a straight line perpendicular to the middle surface. In this system of coordinates Lamé's coefficients are

$$
H_{i}=A_{i}\left(1+\alpha_{3} / R_{i}\right), \quad i=1,2 \quad H_{3}=1,
$$

where $A_{i}=A_{\mathbf{i}}\left(\alpha_{i}\right)$ are the coefficients of the first quadratic form of the middle surface:

$$
d s^{2}=A_{1}^{2} d \alpha_{1}^{2}+A_{2}^{2} d \alpha_{2}^{2} .
$$

Between the parameters $A_{i}$ and the radii of curvature $R_{\imath}$ the Codazzi-Gauss conditions hold:

$$
\begin{gathered}
\left(\frac{A_{2}}{R_{2}}\right)^{\prime}=\frac{A_{2}^{\prime}}{R_{1}}, \quad\left(\frac{A_{1}}{R_{1}}\right)^{\circ}=\frac{A_{1}^{\circ}}{R_{2}}, \quad\left(\frac{A_{2}^{\prime}}{A_{1}}\right)^{\prime}+\left(\frac{A_{2}^{\circ}}{A_{2}}\right)^{\circ}=-\frac{A_{1} A_{2}}{R_{1} R_{2}} \\
\left(\frac{1}{R_{1}}-\frac{1}{R_{2}}\right) A_{1}^{\circ}=-A_{1}\left(\frac{1}{R_{1}}\right)^{\circ}, \quad\left(\frac{1}{R_{1}}-\frac{1}{R_{2}}\right) A_{2}^{\prime}=A_{2}\left(\frac{1}{R_{2}}\right)^{\prime}
\end{gathered}
$$

where ()$^{\prime}=\partial / \partial \alpha_{1},()^{\circ}=\partial / \partial \alpha_{2}$.

Let us now consider the deformation of the shell. The displacements of an arbitrary point $M$ may be defined by three components $u_{1}, u_{2}, u_{3}$. The positive senses of the displacements $u$ follow the directions of the coordinates. The deformation of the shell near the point $M$ is characterized by six components $e_{i j}, i=1,2,3, j=1,2,3$ representing the strains. Between the displacements $u_{i}$ and the strains $e_{i j}$ exist six equations:

$e_{11}=\frac{u_{1}^{\prime}}{H_{1}}+\frac{H_{1}^{\circ} u_{2}}{H_{1}} \frac{1}{H_{2}}+\frac{1}{H_{1}} \frac{\partial H_{1}}{\partial z} u_{3}, \quad e_{12}=\frac{H_{1}}{H_{2}}\left(\frac{u_{1}}{H_{1}}\right)^{\circ}+\frac{H_{2}}{H_{1}}\left(\frac{u_{2}}{H_{2}}\right)^{\prime}, \quad \ldots, \quad e_{33}=\frac{\partial u_{3}}{\partial z}$.

Let us assume that $u_{1}, u_{2}, u_{3}$ may be expressed by the formulae

$$
u_{i}=\left(1+z / R_{1}\right) u+\beta_{1} z, \quad u_{2}=\left(1+z / R_{2}\right) v+\beta_{2} z, \quad u_{3}=w,
$$

where $u\left(\alpha_{\imath}\right), v\left(\alpha_{\imath}\right)$ are the displacements of the middle surface and $\beta_{1}, \beta_{2}$ are the rotation angles of the lateral sides of the shell element during deformation. The strains $e_{i i}$ for $i$, $j=1,2$ may also be written in the following way:

$$
\epsilon_{i j}=\epsilon_{i j}+z \kappa_{i j} .
$$

Introducing the displacements from Eqs. (5) into Eqs. (4), we obtain

$$
\epsilon_{11}=\frac{u^{\prime}}{A_{1}}+\frac{A_{1}^{\circ} v}{A_{1} A_{2}}+\frac{w}{R_{1}}, \quad \epsilon_{12}=\frac{A_{1}}{A_{2}}\left(\frac{u}{A_{1}}\right)^{\circ}+\frac{A_{2}}{A_{1}}\left(\frac{v}{A_{2}}\right)^{\prime}, \cdots,
$$

and

$$
\begin{aligned}
& \kappa_{11}=\frac{\beta_{1}^{\prime}}{A_{1}}+\frac{A_{1}^{\circ} \beta_{2}}{A_{1} A_{2}}-\frac{w}{R_{1}^{2}}+\frac{u}{A_{1}}\left(\frac{1}{R_{1}}\right)^{\prime}+\frac{v}{A_{2}}\left(\frac{1}{R_{1}}\right)^{\circ} \\
& \kappa_{12}=\frac{1}{2}\left[\frac{A_{1}}{A_{2}}\left(\frac{\beta_{1}}{A_{1}}\right)^{\circ}+\frac{A_{2}}{A_{1}}\left(\frac{\beta_{2}}{A_{2}}\right)^{\prime}\right]+\frac{1}{2}\left(\frac{1}{R_{1}}-\frac{1}{R_{2}}\right)\left[\frac{A_{1}}{A_{2}}\left(\frac{u}{A_{1}}\right)^{\circ}-\frac{A_{2}}{A_{1}}\left(\frac{v}{A_{2}}\right)^{\prime}\right], \cdots .
\end{aligned}
$$

2. Stresses and internal forces. The internal forces and moments are the resultants of stresses in the sections of the shell element. For an isotropic material, which is assumed in what follows, we have

$$
\sigma_{11}=\frac{E}{1-\nu^{2}}\left[e_{11}+\nu e_{22}\right]+\frac{\nu}{1-\nu} \sigma_{33}, \quad \tau_{12}=G e_{12} \cdots .
$$


We assume that the distribution of the shear stresses $\tau_{13}$ and $\tau_{23}$ is of the form

$$
\tau_{i 3}=\frac{3}{2} \frac{Q_{i i}}{h}\left[1-\left(\frac{2 z}{h}\right)^{2}\right]\left(1+\frac{z}{R_{j}}\right)^{-1}
$$

which fulfills the conditions $\tau_{13}=\tau_{23}=0$ for $z= \pm h / 2$. From analogy to beams the stress $\sigma_{33}$ is approximated by the equation

$$
\sigma_{33}=\frac{1}{2} Z\left[1+3 \frac{z}{h}-4\left(\frac{z}{h}\right)^{2}\right] .
$$

When $z=+h / 2, \sigma_{3}=Z$ and when $z=-h / 2, \sigma_{3}=0$.

The stress resultants are obtained by integration of stresses across the thickness. We have

$$
\begin{gathered}
N_{i i}=\frac{1}{A_{i}} \int_{-h / 2}^{+h / 2} \sigma_{i i} H_{i} d z, \quad N_{i i}=\frac{1}{A_{i}} \int_{-h / 2}^{+h / 2} \tau_{i i} H_{i} d z \\
M_{i i}=\frac{1}{A_{i}} \int_{-h / 2}^{+h / 2} \sigma_{i i} H_{j} z d z, \quad M_{i j}=\frac{1}{A_{i}} \int_{-h / 2}^{+h / 2} \tau_{i i} H_{j} z d z \\
Q_{i i}=\frac{1}{A_{i}} \int_{-h / 2}^{+h / 2} \tau_{i 3} H_{i} d z .
\end{gathered}
$$

Substituting $\sigma_{i j}$ from Eqs. (9) and using Eqs. (4) and (5), we find, on integration, relations between the internal forces and the moments and the displacements which have a rather complex form. Neglecting the terms $z / R_{1}, z / R_{2}$ as very small in comparison with unity, we obtain simplified relations. Let us adopt them in the form proposed by Novoshilov [17]:

$$
\begin{array}{rlrl}
N_{i i} & =\frac{E h}{1-\nu^{2}}\left(\epsilon_{i i}+v \epsilon_{j i}\right)+\frac{1}{2} \frac{\nu}{1-\nu} Z h & & \\
M_{i i}=D\left(\kappa_{i i}+v \kappa_{i j}\right)+\frac{\nu}{1-\nu} \frac{h^{2}}{10} Z, & M_{i i}=(1-\nu) D \kappa_{i i} \\
N_{i j}=\frac{E h}{2(1+\nu)} \epsilon_{i i}+\frac{1-\nu}{R_{j}} D_{\kappa_{i j}}, & D=\frac{E h^{3}}{12\left(1-\nu^{2}\right)} .
\end{array}
$$

The above relations differ from the relations used in the Donnell-Vlasov theory only by the terms $(1-v) D_{\kappa_{i j}} / R_{i}$ in the expressions for $N_{i j}$. However, these relations enable us to satisfy identically the sixth equation of equilibrium (14) which is impossible assuming $N_{12}=N_{21}$. Moreover, Eqs. (13) have a simple form and are analogous to the corresponding relations in the theory of plates.

Equilibrium of a shell element bounded by the lines $\alpha_{1}=$ const., $\alpha_{2}=$ const. requires the following equations (see Fig. 1):

$$
\begin{gathered}
\left(A_{2} N_{11}\right)^{\prime}-N_{22} A_{2}^{\prime}+\left(A_{1} N_{21}\right)^{\circ}+N_{12} A_{1}^{\circ}+\frac{A_{1} A_{2}}{R_{1}} Q_{11}=0, \\
-\frac{N_{11}}{R_{1}}-\frac{N_{22}}{R_{2}}+\frac{1}{A_{1} A_{2}}\left[\left(A_{2} Q_{11}\right)^{\prime}+\left(A_{1} Q_{22}\right)^{\circ}\right]+Z=0, \\
\left(A_{1} M_{21}\right)^{\circ}+M_{12} A_{1}^{\circ}+\left(A_{2} M_{11}\right)^{\prime}-M_{22} A_{2}^{\prime}-A_{1} A_{2} Q_{11}=0, \cdots, \\
N_{12}-N_{21}+M_{12} / R_{1}-M_{21} / R_{2}=0 .
\end{gathered}
$$



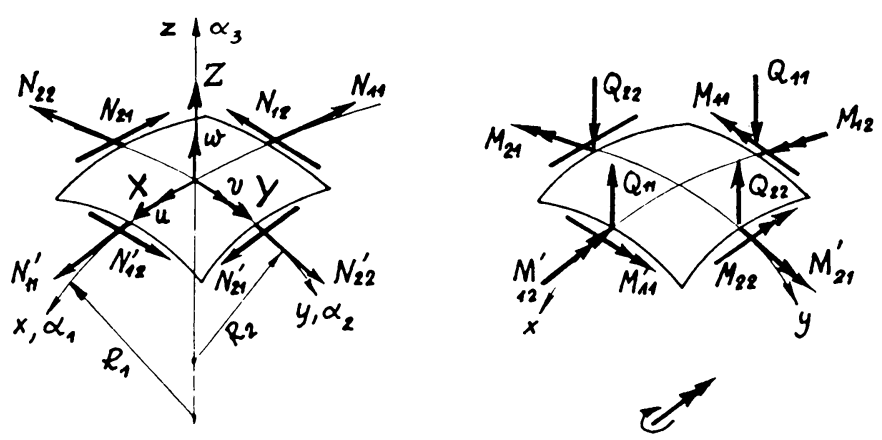

FIG. 1.

3. Effect of transverse shear deformation. In order to find the relations for the determination of the angles $\beta_{1}$ and $\beta_{2}$ we assume that the displacements $u, v, w$ of the middle surface are identical to certain average displacements taken over the thickness of the shell. Reissner made use of Castigliano's principle of least work to introduce the conditions of compatibility and to find additional equations enabling the determination of $\beta_{1}$ and $\beta_{2}$. We define these quantities by equating the work of the resultant couples on the average rotations and the work of the resultant forces on the average displacements $u, v, w$ to the work of the corresponding stresses on the actual displacements $u_{1}$, $u_{2}, u_{3}$ in the same section. I.e., we put

$$
\begin{aligned}
& \frac{1}{A_{2}} \int_{-h / 2}^{+h / 2} \sigma_{11} u_{1} H_{2} d z=N_{11} u+M_{11}\left(\beta_{1}+\frac{u}{R_{1}}\right), \\
& \frac{1}{A_{2}} \int_{-h / 2}^{+h / 2} \tau_{12} u_{1} H_{2} d z=N_{12} u+M_{12}\left(\beta_{1}+\frac{u}{R_{1}}\right), \\
& \frac{1}{A_{2}} \int_{-h / 2}^{+h / 2} \tau_{13} u_{3} H_{2} d z=Q_{11} w, \cdots .
\end{aligned}
$$

Introducing $u_{1}, u_{2}, u_{3}$ and stress resultants into the above equations, we find that all except the last two are fulfilled identically. Substituting $\tau_{13}$ and $\tau_{23}$ from Eqs. (10) in conditions (15) we obtain the average magnitude of the deflection of the shell:

$$
w=\frac{3}{2 h} \int_{-h / 2}^{+h / 2} u_{3}\left[1-\left(\frac{2 z}{h}\right)^{2}\right] d z .
$$

Introducing Eqs. (5) in the last two Eqs. (4) and observing Eq. (9), we find on differentiation

$$
\beta_{i}=\frac{\tau_{i 3}}{G}\left(1+\frac{z}{R_{i}}\right)-\frac{\partial u_{3}}{A_{i} \partial \alpha_{i}} .
$$

Now substituting the shear stresses $\tau_{13}, \tau_{23}$ from Eqs. (10) in the above equations, multiplying both sides of Eqs. (17) by $(3 / 2)\left[1-(2 z / h)^{2}\right](d z / h)$ and integrating between the limits $z= \pm h / z$, we obtain

$$
\beta_{i}=-\frac{\partial w}{A_{i} \partial \alpha_{i}}+\left(\frac{6}{5}-\frac{27}{140} \frac{h^{2}}{R_{i} R_{i}}\right) \frac{Q_{i i}}{h G}
$$


Since $h^{2} / R_{i} R_{i} \ll 1$ it may be neglected in (18). Then the above expressions become identical with those given for plates by Reissner.

4. Compatibility equations. By using Eqs. (7) and (8) and eliminating the displacements $u, v, w$, the following compatibility equations between the strains $\epsilon_{i j}$ and curvatures $\kappa_{i} ;$ may be deduced:

$$
\begin{aligned}
A_{2} \kappa_{22}^{\prime}+A_{2}^{\prime}\left(\kappa_{22}-\kappa_{11}\right)-A_{1} \kappa_{12}^{\circ}-2 A_{1}^{\circ} \kappa_{12} \\
+\frac{1}{R_{2}} A_{1}^{\circ} \epsilon_{12}+\frac{1}{R_{1}}\left[A_{1} \epsilon_{12}^{\circ}+A_{1}^{\circ} \epsilon_{12}-A_{2} \epsilon_{22}^{\prime}-A_{2}^{\prime}\left(\epsilon_{22}-\epsilon_{11}\right)\right] \\
=\frac{12(1+\nu)}{5 E h}\left\{\left(Q_{22}^{\circ}+\frac{A_{2}^{\prime}}{A_{1}} Q_{11}\right)^{\prime}-A_{2}^{\prime}\left(\frac{Q_{11}^{\prime}}{A_{1}}+\frac{A_{1}^{\circ} Q_{22}}{A_{1} A_{2}}\right)\right. \\
\left.\quad-\frac{1}{2}\left[\frac{A_{1}^{2}}{A_{2}}\left(\frac{Q_{11}}{A_{1}}\right)^{\circ}+A_{2}\left(\frac{Q_{22}}{A_{2}}\right)^{\prime}\right]^{\circ}-\frac{1}{2} A_{2}^{\prime}\left[\frac{A_{1}}{A_{2}}\left(\frac{Q_{11}}{A_{1}}\right)^{\circ}+\frac{A_{2}}{A_{1}}\left(\frac{Q_{22}}{A_{2}}\right)^{\prime}\right]\right\}
\end{aligned}
$$

with a second equation following by a change of indexes and a third equation of the form

$$
\begin{aligned}
\frac{\kappa_{11}}{R_{2}}+\frac{\kappa_{22}}{R_{1}}+\frac{1}{A_{1} A_{2}}\left\{\left[\frac{A_{2}}{A_{1}} \epsilon_{22}^{\prime}\right.\right. & \left.+\frac{A_{2}^{\prime}}{A_{1}}\left(\epsilon_{22}-\epsilon_{11}\right)-\frac{1}{2} \epsilon_{12}^{\circ}-\frac{A_{1}^{\circ}}{A_{1}} \epsilon_{12}\right]^{\prime} \\
& \left.+\left[\frac{A_{1}}{A_{2}} \epsilon_{11}^{\circ}+\frac{A_{1}^{\circ}}{A_{2}}\left(\epsilon_{11}-\epsilon_{22}\right)-\frac{1}{2} \epsilon_{12}^{\prime}-\frac{A_{2}^{\prime}}{A_{2}} \epsilon_{12}\right]^{\circ}\right\} \\
= & \frac{12(1+\nu)}{5 E h} \frac{1}{A_{1} A_{2}}\left[\left(\frac{A_{2}}{A_{1} R_{2}} Q_{11}\right)^{\prime}+\left(\frac{A_{1}}{A_{2} R_{1}} Q_{22}\right)^{\circ}\right] .
\end{aligned}
$$

5. Reduction. Now twelve unknown quantities, namely $M_{11}, M_{22}, M_{12}=M_{21}$, $Q_{11}, Q_{22}, N_{11}, N_{22}, N_{12}, N_{21}, w, \beta_{1}, \beta_{2}$, are joined by two equations (18), six equations of equilibrium (14) and finally by three equations of compatibility. In order to transform this set into a form convenient for analysis we introduce a stress function $\Phi$. If the forces $N_{11}, N_{22}, N_{12}, N_{21}$ are expressed by means of the equations

$$
\begin{aligned}
N_{11} & =-\frac{1}{A_{2}}\left(\frac{\phi^{\circ}}{A_{2}}\right)^{\circ}-\frac{A_{2}^{\prime} \phi^{\prime}}{A_{1} A_{2}}-\frac{\phi}{R_{1} R_{2}}-\frac{1}{R_{1}} \int Q_{11} A_{1} d \alpha_{1}-\frac{1-\nu}{R_{1}} D\left(\kappa_{22}-\frac{w}{R_{1} R_{2}}\right) \\
N_{12} & =S+\frac{1-\nu}{R_{2}} D \kappa_{12}, \quad N_{21}=S+\frac{1-\nu}{R_{1}} D \kappa_{12}
\end{aligned}
$$

where

$$
S=\frac{1}{A_{1} A_{2}}\left(\phi^{\prime \circ}-\frac{A_{2}^{\prime} \phi^{\circ}}{A_{2}}-\frac{A_{1}^{\circ} \phi^{\prime}}{A_{1}}\right),
$$

the first two and the last of Eqs. (14) are identically satisfied. On substitution into the first two equations of equilibrium (14) we have

$$
\begin{aligned}
& \left(A_{2} N_{11}\right)^{\prime}-N_{22} A_{2}^{\prime}+\left(A_{1} N_{21}\right)^{\circ}+N_{12} A_{1}^{\circ}+\frac{A_{1} A_{2}}{R_{1}} Q_{11} \\
& =-A_{2} \Phi\left(\frac{1}{R_{1} R_{2}}\right)^{\prime}-A_{2}\left(\frac{1}{R_{1}}+\frac{1}{R_{2}}\right)^{\prime} \int Q_{11} A_{1} d \alpha_{1}+(1-\nu) D F(w) \varphi\left[\left(\frac{1}{R_{i}}\right)^{\prime},\left(\frac{1}{R_{i}}\right)^{\circ}\right] .
\end{aligned}
$$

We see from the above equation that on substitution of Eqs. (21) into the first of Eqs. (14) all the terms containing first-, second- and third-order derivatives of $\phi$ cancel. 
There remains only the term containing the function $\phi$ multiplied by the first-order derivative of the Gauss curvature, and minor terms resulting from the effect of the shear and tangential forces, also multiplied by the derivatives of the radii of curvature. Taking into consideration the slow variation of the curvatures, we find that these terms are insignificant and can be neglected. For constant radii $R_{1}, R_{2}$ these terms vanish and the stress function determined by Eqs. (21) exactly satisfies Eqs. (14a, b).

Expressing in Eq. (14c) the forces $N_{11}, N_{22}, N_{12}, N_{21}$ by means of Eqs. (21) we obtain

$$
\begin{aligned}
& \frac{1}{A_{1} A_{2}}\left[\left(A_{2} Q_{11}\right)^{\prime}+\left(A_{1} Q_{22}\right)^{\circ}\right]=-Z-\Delta_{k} \Phi \\
& \quad-\frac{1}{R_{1}^{2}}\left(\int Q_{11} A_{1} d \alpha_{1}-(1-\nu) D \kappa_{11}\right)-\frac{1}{R_{2}^{2}}\left(\int Q_{22} A_{2} d \alpha_{2}-(1-\nu) D \kappa_{22}\right),
\end{aligned}
$$

where the differential operator $\Delta_{k} \phi$ has the following form:

$$
\Delta_{h} \phi=\frac{1}{A_{1} A_{2}}\left[\left(\frac{A_{2} \phi^{\prime}}{A_{1} R_{2}}\right)^{\prime}+\left(\frac{A_{1} \phi^{\circ}}{A_{2} R_{1}}\right)^{\circ}\right]+\frac{1}{R_{1} R_{2}}\left(\frac{1}{R_{1}}+\frac{1}{R_{2}}\right) \Phi .
$$

Now we shall transform the two equilibrium equations (14d) and (14e), which can be written in a different form. If the bending moments and torques in (14d) are expressed by the relations (13) we obtain the following equation for the force $Q_{11}$ :

$$
Q_{11}=\frac{D}{A_{1}}\left(\kappa_{11}+\kappa_{22}\right)^{\prime}-\frac{1-\nu}{A_{1} A_{2}}\left[\left(A_{2} \kappa_{22}\right)^{\prime}-A_{2}^{\prime} \kappa_{11}-\left(A_{1} \kappa_{12}\right)^{\circ}-A_{1}^{\circ} \kappa_{12}\right]+\frac{h^{2}}{10} \frac{\nu}{1-\nu} \frac{Z^{\prime}}{A_{1}} .
$$

The expressions in (25) contained in square brackets can be simplified by means of the first two identities (19). Remembering that for $u=v=0, \epsilon_{11}=w / R_{1}, \epsilon_{22}=w / R_{2}$, $\boldsymbol{\epsilon}_{12}=0$, and making use of Eq. (8) and the Codacci-Gauss conditions (3), we obtain the following equation:

$$
\begin{aligned}
& \frac{1}{A_{1} A_{2}}\left[\left(A_{2} K_{22}\right)^{\prime}-A_{2}^{\prime} \kappa_{11}-\left(A_{1} \kappa_{12}\right)^{\circ}-A_{1}^{\circ} \kappa_{12}\right] \\
& =\frac{w^{\prime}}{A_{1} R_{1} R_{2}}+\frac{6(1+\nu)}{5 \operatorname{Lh} A_{1} A_{2}}\left\{Q_{22}^{\prime \circ}-\left(\frac{A_{1} Q_{11}^{\circ}}{A_{2}}\right)^{\circ}+\left(\frac{A_{2}^{\prime}}{A_{1}}\right)^{\prime} Q_{11}+\frac{A_{1}^{\circ}}{A_{1} A_{2}}\left[A_{1}^{\circ} Q_{11}-\left(A_{2} Q_{22}\right)^{\prime}\right]\right\} .
\end{aligned}
$$

We obtain the second equation by a simple change of indexes and derivatives. On the basis of Eqs. (8) and (18),

$$
\begin{gathered}
\kappa_{11}+\kappa_{22}=-\Delta w-\left(\frac{1}{R_{1}^{2}}+\frac{1}{R_{2}^{2}}\right) w+\frac{12(1+\nu)}{5 E h} \frac{1}{A_{1} A_{2}}\left[\left(A_{2} Q_{11}\right)^{\prime}+\left(A_{1} Q_{22}\right)^{\circ}\right] \\
\Delta w=\frac{1}{A_{1} A_{2}}\left[\left(\frac{A_{2} w^{\prime}}{A_{1}}\right)^{\prime}+\left(\frac{A_{1} w^{\circ}}{A_{2}}\right)^{\circ}\right],
\end{gathered}
$$

where $\Delta$ is the Laplacian operator. The terms in Eqs. (8) containing the derivatives of the radii of curvatures, such as $u / A_{1}\left(1 / R_{i}\right)^{\prime}$, are here neglected.

Through using the equation of equilibrium (23) we have

$$
\begin{aligned}
\kappa_{11}+\kappa_{22}= & -\Delta w-\left(\frac{1}{R_{1}^{2}}+\frac{1}{R_{2}^{2}}\right) w-\frac{12(1+\nu)}{E h}\left[Z+\Delta_{k} \phi\right] \\
& +\frac{h^{2}}{5(1-\nu)}\left[\left(\frac{1}{R_{1}^{2}}+\frac{1}{R_{2}^{2}}\right) \Delta w-(1-\nu)\left(\frac{\kappa_{11}}{R_{1}^{2}}+\frac{\kappa_{22}}{R_{2}^{2}}\right)\right] .
\end{aligned}
$$


As the underlined term of the order $h^{2} / R^{2}$ is much smaller than the first term on the right hand-side of Eq. (28) it will be neglected in what follows.

Now, introducing Eq. (26) and Eq. (28) into Eq. (25), we obtain the following equation for the shearing force $Q_{11}$ :

$$
\begin{aligned}
Q_{11}- & \frac{h^{2}}{10}\left\{\Delta Q_{11}-\frac{1}{A_{1}^{2} A_{2}^{2}}\left[\left(A_{1}^{\circ}\right)^{2}+\left(A_{2}^{\prime}\right)^{2}\right] Q_{11}\right. \\
& \left.-\frac{2 A_{2}^{\prime}}{A_{1} A_{2}^{2}} Q_{22}^{\circ}+\frac{2 A_{1}^{\circ}}{A_{1}^{2} A_{2}} Q_{22}^{\prime}-\frac{1}{A_{1} A_{2}}\left[\left(\frac{A_{2}^{\prime}}{A_{2}}\right)^{\circ}-\left(\frac{A_{1}^{\circ}}{A_{1}}\right)^{\prime}\right] Q_{22}\right\} \\
= & -\frac{D}{A_{1}}\left[\left(\Delta+\frac{1}{R_{1}^{2}}+\frac{1-\nu}{R_{1} R_{2}}+\frac{1}{R_{2}^{2}}\right) w\right]^{\prime}-\frac{h^{2}}{10(1-\nu) A_{1}}\left[Z+(1+\nu) \Delta_{k} \phi\right]^{\prime} .
\end{aligned}
$$

The second equation for $Q_{22}$ is similar and may be obtained by an interchange of the indexes 1 and 2. The three Eqs. (29a, b) and (22) are a set of differential equations containing four unknown functions $w, \phi, Q_{11}$ and $Q_{22}$. In order to have a complete set of equations one more equation is necessary. This additional relation between the same functions may be obtained from the third condition of compatibility. Without describing in detail these manipulations, since they are entirely analogous to those involved in the deduction of Eqs. (22), (29), etc., the final result may be given in the form

$$
\begin{aligned}
\frac{1}{E h} \Delta\left(\Delta+\frac{2}{R_{1} R_{2}}\right) \phi & +\Delta_{k} w-\frac{D}{E h}-\frac{1}{A_{1} A_{2}}\left\{\frac{1}{A_{1}}\left[A_{2}\left(\frac{2-\nu}{R_{2}}-\frac{\nu}{R_{1}}\right) \kappa_{11}+\left(\frac{1}{R_{2}}-\frac{\nu(2-\nu)}{R_{1}}\right) \kappa_{22}\right]^{\prime}\right. \\
& \left.-\frac{A_{2}^{\prime}}{A_{1}}\left[\left(\frac{1}{R_{1}}-\frac{\nu(2-\nu)}{R_{2}}\right) \kappa_{11}+\left(\frac{2-\nu}{R_{1}}-\frac{\nu}{R_{2}}\right) \kappa_{22}\right]\right\}^{\prime} \\
& -\frac{D}{E h} \frac{1}{A_{1} A_{2}}\left\{\frac{1}{A_{2}}\left[A_{1}\left(\frac{1}{R_{1}}-\frac{\nu(2-\nu)}{R_{2}}\right) \kappa_{11}+\left(\frac{2-\nu}{R_{1}}-\frac{\nu}{R_{2}}\right) \kappa_{22}\right]^{\circ} \quad\right. \\
& \left.-\frac{A_{1}^{\circ}}{A_{2}}\left[\left(\frac{2}{R_{2}}-\frac{\nu}{R_{1}}\right) \kappa_{11}+\left(\frac{1}{R_{2}}-\frac{\nu(2-\nu)}{R_{1}}\right) \kappa_{22}\right]\right\}^{\circ} \\
& \frac{1}{E h A_{1} A_{2}}\left\{\left[\frac{A_{2}}{A_{1}} \phi\left(\frac{1}{R_{1} R_{2}}\right)^{\prime}\right]^{\prime}+\left[\frac{A_{1}}{A_{2}} \phi\left(\frac{1}{R_{1} R_{2}}\right)^{\circ}\right]\right]^{\circ}=-\frac{\nu}{2 E} \Delta Z .
\end{aligned}
$$

In deriving the above equation certain minor terms of the order $h^{2} / R^{2}$ resulting from the effect of transverse shear deformation have been neglected. However, this equation may be simplified still further. We may neglect the terms including the derivatives of the Gauss curvature, since for shells for slowly varying curvatures they are of minor importance. In order to simplify the above equation we may also neglect the terms proportional to $-D / E h$, which are of minor importance. Adding a very small term $\phi / E h R_{1}^{4} R_{2}^{4}$ we obtain the following equation:

$$
\frac{1}{E h}\left(\Delta+\frac{1}{R_{1} R_{2}}\right)^{2} \phi+\Delta_{k} w=-\frac{\nu}{2 E} \Delta Z .
$$

The term $-\nu \Delta Z / 2 E$ on the right-hand side of Eq. (31) represents the effect of the stress $\sigma_{33}$ produced by the load perpendicular to the shell surface. Usually it is small and only in the case of concentrated loads may it be larger. The set of four equations (22), (29a, b), (31) includes four unknown functions $w, \phi, Q_{11}, Q_{22}$ and enables one to solve the problem of an arbitrary shell taking into account the effect of transverse shear and normal 
stresses. It is possible to eliminate the shear forces from Eq. (22). Then we obtain a set of only two equations which includes only two unknown functions $w$ and $\phi$. Substituting the shear forces $Q_{11}$ and $Q_{22}$ from Eqs. $(29 \mathrm{a}, \mathrm{b})$ into Eq. (22) we get, after some manipulation:

$$
\begin{array}{r}
D\left(\Delta+\frac{1}{R_{1}^{2}}+\frac{1}{R_{2}^{2}}\right)^{2} w-\left(1-\frac{h^{2}}{5(1-\nu)} \Delta\right) \Delta_{k} \phi+(1-\nu) D\left[\left(\frac{1}{R_{1}}-\frac{1}{R_{2}}\right)\left(\frac{\kappa_{11}}{R_{1}}-\frac{\kappa_{22}}{R_{2}}\right)\right. \\
\left.+\frac{1}{R_{1} R_{2}}\left(\frac{1}{R_{1}^{2}}+\frac{1}{R_{2}^{2}}\right) w\right]=\left[1-\frac{2-\nu}{1-\nu} \frac{h^{2}}{10} \Delta\right] Z .
\end{array}
$$

In order to simplify the above equation we neglect the underlined term which is of minor importance. Then we have the second simple equation relating the functions $w$ and $\phi$

$$
D\left(\Delta+\frac{1}{R_{1}^{2}}+\frac{1}{R_{2}^{2}}\right)^{2} w-\left(1-\frac{h^{2}}{5(1-\nu)} \Delta\right) \Delta_{k} \phi=\left[1-\frac{2-\nu}{1-\nu} \frac{h^{2}}{10} \Delta\right] Z .
$$

Neglecting in Eqs. (31) and (33) the terms which contain the radii $R_{1}$ and $R_{2}$ (except $\Delta_{k}$ ) and introducing the system of Cartesian coordinates, we get the equations for shallow shells similar to those obtained earlier by Naghdi [10]. The difference between these equations results only from having here taken into account the effect of the transverse normal stress $\sigma_{33}$. This effect is of the same order as the effect of the transverse shear deformation.

The equations (29), (31), and (33) are together a twelve-order system. However, this system should be of the tenth order since at every edge we have only five boundary conditions. In case of Cartesian coordinates and shallow shells the further reduction may easily be performed. When $A_{1}=A_{2}=$ const., Eqs. (29) imply the second-order equation

$$
A_{1} Q_{11}^{\circ}-A_{2} Q_{22}^{\prime}=\frac{h^{2}}{10} \Delta\left(A_{1} Q_{11}^{\circ}-A_{2} Q_{22}^{\prime}\right)
$$

or

$$
\Delta \psi-\frac{10}{h^{2}} \psi=0
$$

where

$$
\psi=A_{1} Q_{11}^{\circ}-A_{2} Q_{22}^{\prime} .
$$

Now we have a tenth-order system for the three dependent variables $w, \phi$ and $\psi$. The shear forces $Q_{11}$ and $Q_{22}$ may be expressed as a combination of derivatives of these three:

$$
\begin{aligned}
& Q_{11}=-D \frac{1}{A_{1}}(\Delta w)^{\prime}-\frac{h^{2}}{10(1-\nu) A_{1}}\left[(2-\nu) Z+2 \Delta_{k} \phi\right]^{\prime}+\frac{h^{2}}{10} \frac{\psi^{\circ}}{A_{2}}, \\
& Q_{22}=-D \frac{1}{A_{2}}(\Delta w)^{\circ}-\frac{h^{2}}{10(1-\nu) A_{2}}\left[(2-\nu) Z+2 \Delta_{k} \phi\right]^{\circ}-\frac{h^{2}}{10} \frac{\psi^{\prime}}{A_{1}} .
\end{aligned}
$$

It is interesting to compare the above Eqs. (31) and (33) with those obtained by other authors. Setting $R_{1}=R_{2}=R$ we have the case of the spherical shell. If we neglect the effect of transverse shear deformation Eqs. (31) and (33) are equivalent to the equations for spherical shells obtained by Vlasov [4]. In the case of cylindrical shells, for which 
$R_{1}=\infty$ and $R_{2}=R$, Eqs. (31) and (33) may be reduced to Morley's equation [14] (see also [15]).

\section{REFERENCES}

[1] L. H. Donnell, Stability of thin-walled tubes under torsion, NACA Report \#479, 1933

[2] M. Musthari, Some generalizations of the theory of thin shells, Izv. Fiz. Mat. O. Va. Kazan. Univ. (8) 11 (1938)

[3] K. Marguerre, Zur Theorie der gekrummten Platte grosser Formanderung, Proc. Fifth Internat. Congress Applied Mechanics Cambridge, Mass., 1938, pp. 93-101

[4] V. Z. Vlasov, General theory of shells and its applications in technology, Gittl, Moscow, 1949 (Russian)

[5] S. Lukasiewicz, Supplement to the technical theory of shells, Rozprawy Inż. 11, (1963)

[6] F. B. Hildebrand, E. Reissner and G. B. Thomas, Notes on the foundations of the theory of small displacements of orthotropic shells, NACA Technical Notes \#1833, 1949

[7] A. E. Green and W. Zerna, Theoretical elasticity, Clarendon Press, Oxford, 1954

[8] E. Reissner, Stress-strain relations in the theory of thin elastic shells, J. Math. Physics 31, 109-119 (1952)

[9] E. Reissner, Proc. Amer. Soc. Civ. Eng. EM 23, 1962

[10] P. M. Naghdi, Note on the equations of shallow elastic shells, Quart. Appl. Math. 14, 331-333 (1956)

[11] P. M. Naghdi, On the theory of thin elastic shells, Quart. Appl. Math. 14, 369-380 (1957)

[12] J. P. Wilkinson and A. Kalnins, On nonsymmetric dynamic problems of elastic spherical shells, J. Appl. Mech. 32, 525 (1965)

[13] J. P. Wilkinson and A. Kalnins, Deformation of open spherical shells under arbitrarily located concentrated loads, J. Appl. Mech. 33, 305-312 (1966)

[14] L. S. D. Morley, An improvement on Donnell's approximation for thin-walled circular cylinders, Quart. J. Mech. Appl. Math. 12, 89-99 (1959)

[15] S. Lukasiewicz, The equations of the theory of non-shallow thin shells, Arch Bud. Masz. 12 (1965)

[16] S. Kukasiewicz, On the technical theory of non-shallow shells, Arch. Bud. Masz. 15 (1968) 